\title{
Tropism of influenza B viruses in human respiratory tract explants and airway organoids
}

\author{
Christine H.T. Bui ${ }^{1,4}$, Renee W.Y. Chan ${ }^{2,4}$, Mandy M.T. Ng ${ }^{1}$, M-C. Cheung ${ }^{1}$, \\ Ka-chun Ng (i) ${ }^{1}$, Megan P.K. Chan ${ }^{1}$, Louisa L.Y. Chan ${ }^{1,2}$, Joanne H.M. Fong ${ }^{1}$, \\ J.M. Nicholls ${ }^{3}$, J.S. Malik Peiris ${ }^{1}$ and Michael C.W. Chan ${ }^{1}$
}

Affiliations: ${ }^{1}$ School of Public Health, Li Ka Shing Faculty of Medicine, The University of Hong Kong, Hong Kong SAR, China. ${ }^{2}$ Dept of Paediatrics, Faculty of Medicine, The Chinese University of Hong Kong, Hong Kong SAR, China. ${ }^{3}$ Dept of Pathology, Queen Mary Hospital, Li Ka Shing Faculty of Medicine, The University of Hong Kong, Hong Kong SAR, China. ${ }^{4}$ Joint first authors.

Correspondence: Michael C.W. Chan, School of Public Health, Li Ka Shing Faculty of Medicine, The University of Hong Kong, L6-39, 6/F, Laboratory Block, Faculty of Medicine Building, 21 Sassoon Road, Pokfulam, Hong Kong SAR, China. E-mail: mchanahku.hk

@ERSpublications

Influenza B viruses can infect and replicate in various human respiratory tract cell types, with higher preference for the conducting airway than the lower lung, and with similar tropism and replication competence as human seasonal influenza $\mathrm{A}$ viruses bit.ly/2Ys3LkD

Cite this article as: Bui CHT, Chan RWY, Ng MMT, et al. Tropism of influenza B viruses in human respiratory tract explants and airway organoids. Eur Respir J 2019; 54: 1900008 [https://doi.org/10.1183/ 13993003.00008-2019].

ABSTRACT Despite causing regular seasonal epidemics with substantial morbidity, mortality and socioeconomic burden, there is still a lack of research into influenza B viruses (IBVs). In this study, we provide for the first time a systematic investigation on the tropism, replication kinetics and pathogenesis of IBVs in the human respiratory tract.

Physiologically relevant ex vivo explant cultures of human bronchus and lung, human airway organoids, and in vitro cultures of differentiated primary human bronchial epithelial cells and type-I-like alveolar epithelial cells were used to study the cellular and tissue tropism, replication competence and induced innate immune response of 16 IBV strains isolated from 1940 to 2012 in comparison with human seasonal influenza A viruses (IAVs), H1N1 and H3N2. IBVs from the diverged Yamagata- and Victoria-like lineages and the earlier undiverged period were included.

The majority of IBVs replicated productively in human bronchus and lung with similar competence to seasonal IAVs. IBVs infected a variety of cell types, including ciliated cells, club cells, goblet cells and basal cells, in human airway organoids. Like seasonal IAVs, IBVs are low inducers of pro-inflammatory cytokines and chemokines. Most results suggested a higher preference for the conducting airway than the lower lung and strain-specific rather than lineage-specific pathogenicity of IBVs.

Our results highlighted the non-negligible virulence of IBVs which require more attention and further investigation to alleviate the disease burden, especially when treatment options are limited. 


\section{Introduction}

Influenza B virus (IBV) was first isolated in 1940 [1]. It belongs to the family Orthomyxoviridae, is enveloped and contains an eight-segment negative-sense single-stranded RNA genome [2]. Since the mid-1970s, IBVs have diverged into Yamagata-like and Victoria-like lineages according to the phylogenetic and antigenic characteristics of their haemagglutinin (HA) surface glycoproteins [3]. They have been co-circulating worldwide causing regular seasonal epidemics with substantial morbidity, mortality and socioeconomic burden. Approximately once every 3 years, IBVs are the predominant circulating strain of influenza virus with prevalence as high as $70 \%$ [4]. In the United States, estimated rates of influenza-associated hospitalisations were second only to A/H3N2, higher than A/H1N1 [5]. Pneumonia, respiratory failure and acute respiratory distress syndrome (ARDS) associated with IBV infection may lead to death [6]. Of all influenza-associated excess mortality in the US, IBVs on average contribute to 29\%, their contribution sometimes reaching as high as 51-95\% [7]. In Hong Kong, an average hospitalisation cost of USD 1328.33 was attributed to each IBV-infected child [8].

While IBVs share major structural similarities with influenza A viruses (IAVs), they are evolutionarily diverged and genetically incompatible, with no intertypic reassortment with IAVs detected in nature [2]. However, research on IBVs is still overshadowed by that focused on IAVs. IBV tropism and pathogenesis are still poorly studied.

The ability of influenza virus to replicate in human upper and conducting airways is often associated with its transmissibility, so viral infection of ex vivo explant cultures of human nasopharynx and bronchus has been listed by the World Health Organization (WHO) as a parameter for assessing transmissibility [9]. Recently, we showed that human airway organoids represented an alternative physiologically relevant model of human ex vivo bronchus cultures, with the advantages of being more abundantly available and more easily manipulated for co-staining with defined cellular markers to study cellular tropism [10]. Replication in the lower lung is often associated with disease severity and ex vivo explant cultures of human lung have been used extensively by our group to investigate viral pathogenesis [11-13].

Our study provides for the first time a systematic investigation into tissue tropism and replication competence of IBVs in ex vivo explant cultures of human bronchus and lung and human airway organoids using a panel of 16 representative IBV strains from 1940 to 2012, including viruses from both Yamagataand Victoria-like lineages as well as a virus from the period prior to the divergence of these two lineages. Innate immune responses induced by IBVs were studied in in vitro cultures of differentiated primary human bronchial epithelial cells (dHBECs) and primary human type-I-like alveolar epithelial cells (AECs). In parallel, human seasonal $\mathrm{A} / \mathrm{H} 1 \mathrm{~N} 1$ and $\mathrm{A} / \mathrm{H} 3 \mathrm{~N} 2$ were used for comparison.

\section{Material and methods}

Technical details are presented in the supplementary materials.

\section{Viruses}

Virus stocks of 16 IBVs and five IAVs (table 1) were prepared in Madin-Darby canine kidney (MDCK) cells or specific-pathogen-free embryonated chicken eggs with limited passage. Viral titres were determined using median tissue culture infective dose (TCID50) and plaque assays.

\section{Infection of ex vivo explant cultures of human bronchus and lung}

Fresh human bronchus and lung were collected from non-tumour residual tissues of patients undergoing surgical resection with informed consent. Approval was granted by the institutional review board of the University of Hong Kong and the Hospital Authority (Hong Kong West) (approval no: UW 14-119). Tissues were cultured and infected in $\sim 10^{6} \mathrm{TCID} 50 \cdot \mathrm{mL}^{-1}$ virus for $1 \mathrm{~h}$ at $37^{\circ} \mathrm{C}$ as previously described [10-13]. Supernatant was harvested at 1,24 and $48 \mathrm{~h}$ post-infection (hpi) for virus titration by TCID50 assay. Tissues were fixed in $10 \%$ neutral-buffered formalin at 48 hpi for immunohistochemical staining.

\section{Infection of human airway organoids}

Human airway organoids were cultured from cells isolated from non-tumour lung tissue and infected in $\sim 10^{6} \mathrm{TCID}_{50} \cdot \mathrm{mL}^{-1}$ virus for $1 \mathrm{~h}$ at $37^{\circ} \mathrm{C}$ as described [10]. Supernatant was harvested at 1,24 and $48 \mathrm{hpi}$ for virus titration by TCID50 assay. Total virus released from 1 to $48 \mathrm{hpi}$ was represented by the area under the curve (AUC) from viral titre plots. Organoids were fixed in $4 \%$ paraformaldehyde at 24 and 48 hpi for immunohistochemical double staining.

\section{Infection of in vitro human primary cell cultures}

dHBECs [13,14] and AECs [11-13] were isolated, cultured and infected at a multiplicity of infection (MOI) of 0.01 or 2 for $1 \mathrm{~h}$ at $37^{\circ} \mathrm{C}$. Supernatant was harvested at $1,24,48,72$ and 96 hpi for virus titration 
TABLE 1 List of influenza $A$ and $B$ viruses (IAVs and IBVs, respectively) used in this study

IBV-Yamagata ${ }^{\#}$

IBV-Victoria?

Human seasonal IAVs
Undiverged IBV

B/Lee/1940

B/Harbin/7/1994

B/Yamanashi/166/1998

B/Taiwan/N1902/2004

B/HongKong/84329/2005

B/Florida/4/2006

B/HongKong/412 407/2008

B/HongKong/448 799/2012

B/Victoria/2/1987

B/Shandong/7/1997

B/Taiwan/N1706/2002

B/Tehran/80/2002

B/Malaysia/2506/2004

B/HongKong/38551/2005

B/HongKong/204133/2006

B/HongKong/407373/2011

A/HongKong/54/1998 (H1N1)

A/Oklahoma/447/2008 (H1N1)

A/HongKong/1174/1999 (H3N2)

A/Oklahoma/370/2005 (H3N2)

Highly pathogenic avian influenza (HPAI) H5N1

A/Hong Kong/483/1997

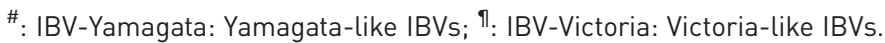

by TCID50 assay. Total virus released from 1 to 96 or 24 to 96 hpi was represented by AUC from viral titre plots. Cell lysates were collected at 6 or 8 and 24 hpi for measurement of mRNA expression. Except when infecting mucus-containing dHBECs, all cells were washed with PBS, pH 7.4, prior to infection.

Mucus content was measured in our earlier dHBEC cultures using an enzyme-linked lectin assay (ELLA) with horseradish peroxidase conjugated wheat germ agglutinin (HRP-WGA) which specifically binds sialic acids and monomers and oligomers of $\mathrm{N}$-acetyl-D-glucosamine. Mucins were quantified as they comprise a significant portion of airway mucus and are rich in sialic acids and N-acetyl-D-glucosamine. 200-300 $\mu \mathrm{L}$ of PBS was added to the apical compartment of a 24-well transwell insert for $20 \mathrm{~min}$ to rinse out the mucus secreted by the dHBECs. ELLA results showed that washing with PBS can remove mucus effectively. $\sim 4000-8000 \mu \mathrm{g} \cdot \mathrm{mL}^{-1}$ of mucins were present in this setting.

\section{Immunohistochemical staining}

Paraffin-embedded human tissues and airway organoids were single stained with IBV- or IAV-specific antibody and double-stained with IBV- or IAV-specific antibody and cell-type specific markers acetyl- $\alpha$-tubulin, SCGB1A1/CC10, MUC5AC or p63- $\alpha$, respectively, as described previously [10, 11].

Cytokine and chemokine and interferon-stimulated gene measurement

mRNA expression of cytokines and chemokines in dHBECs and AECs was measured by real-time PCR as described previously [11-13].

\section{Desialylation-haemagglutination assay}

The effect of desialylation on IBV and IAV haemagglutination of Turkey red blood cells (TRBCs) was studied as described previously [11]. Untreated TRBCs were compared with those treated with Glyko Sialidase S (Prozyme, Hayward, CA, USA) or sialidase DAS181 (NexBio, San Diego, CA, USA).

Data analysis

Viral replication was categorised as productive replication, marginal replication or non-replication with mean titres at $24 \mathrm{hpi}$ or later being $\geqslant 10^{2.5} \mathrm{TCID} 50 \cdot \mathrm{mL}^{-1}(\geqslant 1 \log$ higher than the TCID50 assay detection limit of $10^{1.5}$ TCID $\left.50 \cdot \mathrm{mL}^{-1}\right)$, between $10^{2}$ and $<10^{2.5}$ TCID $50 \cdot \mathrm{mL}^{-1}(0.5$ to $<1$ log higher than the detection limit) and $<10^{2} \mathrm{TCID} 50 \cdot \mathrm{mL}^{-1}(<0.5 \log$ higher than the detection limit), respectively.

Statistical analysis was performed using GraphPad Prism version 5 (San Diego, CA, USA). Viral replication kinetics and mRNA expressions between experimental groups at different time points were compared by two-way ANOVA, while AUC values were compared by one-way ANOVA, both with Bonferroni post-tests. 


\section{Results}

IBVs replicated productively in human bronchial explants

The majority (15 out of 16) of IBV strains tested replicated productively in explant cultures of human bronchus (figure 1a). Yamagata-like B/Taiwan/N1902/2004, B/Florida/4/2006, B/HongKong/412 407/2008 and B/HongKong/448799/2012 and Victoria-like B/Malaysia/2506/2004, B/HongKong/38551/2005 and $\mathrm{B} /$ HongKong/407373/2011 replicated most efficiently (mean peak titres $\geqslant 10^{5} \mathrm{TCID} 50 \cdot \mathrm{mL}^{-1}$ ), being comparable to those of seasonal A/H1N1 (A/Oklahoma/447/2008) and A/H3N2 (A/Oklahoma/370/2005), and significantly higher than those of Yamagata-like B/Harbin/7/1994, Victoria-like B/Victoria/2/1987, B/ Taiwan/N1706/2002 and B/HongKong/204133/2006, and the early B/Lee/1940 strains (figure 1a and supplementary figure S1). Most IBVs and seasonal IAVs exhibited similar replication patterns with 24 hpi peaks. Additional information on the replication kinetics of seasonal A/H1N1 (A/HongKong/54/1998) and A/H3N2 (A/HongKong/1174/1999) is provided in supplementary figure S3a. Immunohistochemical staining for IBV nucleoprotein showed ciliated and non-ciliated epithelial cells in explant cultures of human bronchus infected with IBV (figure $1 \mathrm{~b}$ and supplementary figure S4a). The extensiveness of IBV nucleoprotein expression was correlated with viral replication competence with extensive clusters of infected cells observed in highly replicating strains. Furthermore, sloughing and thinning of the epithelium was observed in IBV-infected explants.

\section{Cellular tropism of IBVs in human airway organoids}

To further understand the tropism of IBVs in human conducting airways, human airway organoids were infected with Yamagata-like B/Taiwan/N1902/2004 and Victoria-like B/HongKong/38551/2005 in comparison with seasonal A/H1N1 (A/Oklahoma/447/2008) and A/H3N2 (A/Oklahoma/370/2005). All viruses showed highly productive replication as in human bronchial explants with mean peak titres being $>10^{5} \mathrm{TCID} 50 \cdot \mathrm{mL}^{-1}$ at $48 \mathrm{hpi}$ (figure $2 \mathrm{a}$ ). AUC analysis from 1 to $48 \mathrm{hpi}$ demonstrated similar overall replication between the two IBVs and A/Oklahoma/370/2005, which were significantly lower than A/Oklahoma/447/2008 (figure 2b). Immunohistochemical double staining revealed both IBVs and seasonal IAVs infected acetyl- $\alpha$-tubulin ${ }^{+}$ciliated cells, SCGB1A1 $1^{+} / \mathrm{CC} 10^{+}$secretory club cells, MUC5AC ${ }^{+}$secretory goblet cells and p63- $\alpha^{+}$basal cells (figure $2 \mathrm{c}$ and supplementary figure S4c). Additional information on the replication kinetics of seasonal A/H1N1 (A/HongKong/54/1998) and A/H3N2 (A/HongKong/1174/ 1999) in human airway organoids and the corresponding immunohistochemical double staining is provided in supplementary figure S3c and d, respectively.

\section{IBVs replicated productively in human lung explants}

Using the same virus panel as in human bronchus, most (13 out of 16) IBVs replicated productively in explant cultures of human lung (figure 3a). Nonetheless, mean viral titres of IBVs and seasonal IAVs (A/Oklahoma/447/2008 and A/Oklahoma/370/2005) were mostly $\leqslant 10^{4} \mathrm{TCID} 50 \cdot \mathrm{mL}^{-1}$ at 24 and $48 \mathrm{hpi}$ as opposed to those in human bronchial explants, except for Victoria-like B/Malaysia/2506/2004, and mostly comparable (figure 3a and supplementary figure S2). As in human bronchus, most IBVs and seasonal IAVs produced the highest titres at $24 \mathrm{hpi}$. Additional information on the replication kinetics of seasonal A/H1N1 (A/HongKong/54/1998) and A/H3N2 (A/HongKong/1174/1999) is provided in supplementary figure S3b. Immunohistochemical staining for IBV nucleoprotein stained positive in alveolar epithelial cells (figure $3 \mathrm{~b}$ and supplementary figure S4b). Explants infected with marginal and non-replicating strains correspondingly showed no or a few sparsely located positive cells, while those infected with productive replicating strains generally had more positive cells.

\section{IBV-induced innate immune response in dHBECs}

Among the highest replicating IBV strains in human bronchial explants, Yamagata-like B/Taiwan/N1902/ 2004 and B/HongKong/448799/2012 and Victoria-like B/HongKong/38551/2005 and B/HongKong/407 $373 / 2011$ were evaluated for their replication efficiency and induction of innate immune response in dHBECs in comparison to seasonal A/H1N1 (A/Oklahoma/447/2008) and A/H3N2 (A/Oklahoma/370/ 2005). At MOI 0.01 , all viruses showed highly productive replication (mean peak titres $\geqslant 10^{6} \mathrm{TCID} 50 \cdot \mathrm{mL}^{-1}$ ) (figure 4a). AUC analysis from 1 to $96 \mathrm{hpi}$ indicated comparable overall replication between the four IBVs with only B/HongKong/448799/2012 having lower AUC than B/HongKong/38551/2005 (figure 4b). However, all IBVs exhibited significantly lower AUC than the two seasonal IAVs. At MOI 2, there was a trend suggesting higher induction of pro-inflammatory cytokines and chemokines interferon (IFN)- $\beta$, $\mathrm{C}-\mathrm{X}-\mathrm{C}$ motif chemokine (CXCL) 10 and $\mathrm{C}-\mathrm{C}$ motif chemokine ligand (CCL) $5 \mathrm{mRNA}$ by IBVs than seasonal IAVs at $24 \mathrm{hpi}$, with statistical significance in the case of Yamagata-like IBVs (figure 4c).

\section{Inhibitory effect of mucus on replication of IBVs}

Airway mucus constantly lines the respiratory tract and can act as a barrier to trap pathogens and prevent them from infecting the underlying epithelium [15]. As the mucin barrier potentially has an important 

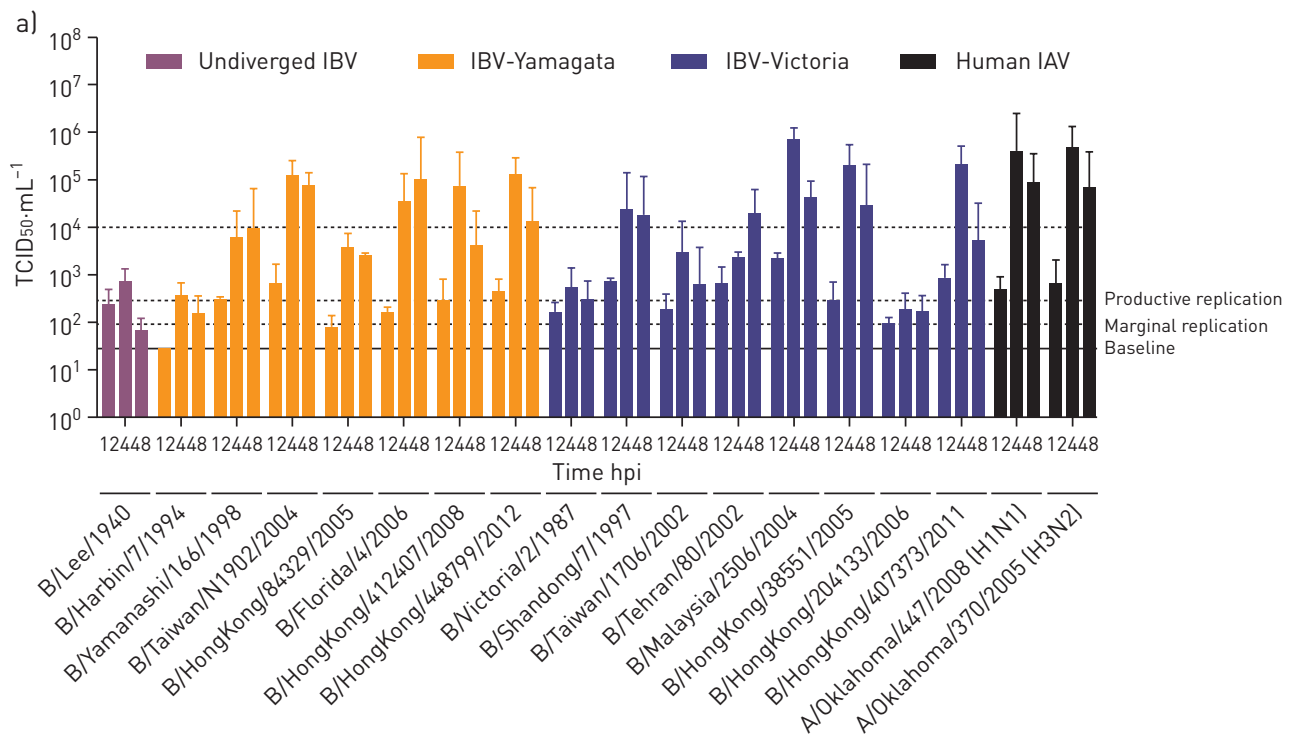

b)
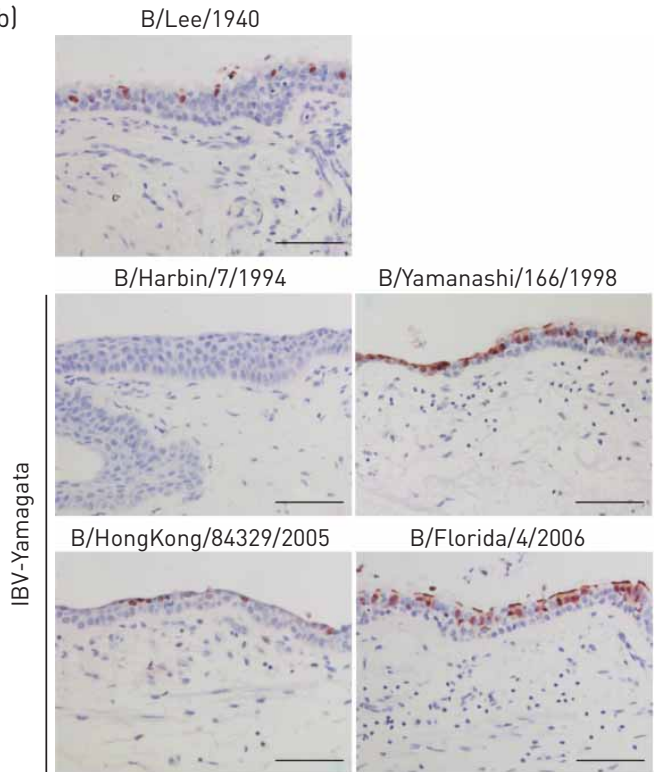

B/Taiwan/N1902/2004

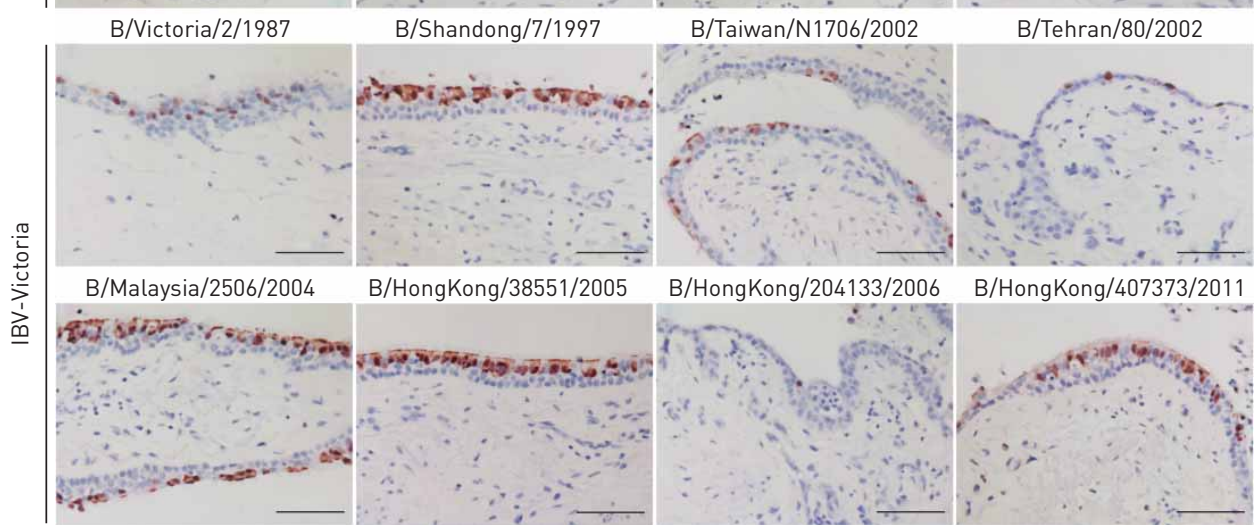

FIGURE 1 a) Replication kinetics of human seasonal influenza A and B viruses (IAVs and IVBs, respectively) in explant cultures of human bronchus infected with $\sim 10^{6} \mathrm{TCID} 50 \cdot \mathrm{mL}^{-1}$ at $37^{\circ} \mathrm{C}$. Viral titres in culture medium (mean \pm SEM, $n \geqslant 3$ ) were determined by median tissue culture infective dose (TCID50) assay with a detection limit of $10^{1.5} \mathrm{TCID} 50 \cdot \mathrm{mL}^{-1}$ denoted by the baseline. Statistical significance between viral titres at different times post-infection was calculated by two-way ANOVA with Bonferroni post-tests (supplementary figure S1). b) Representative images of immunohistochemical staining for IBV nucleoprotein (brown) in virus-infected explant cultures of human bronchus at $48 \mathrm{~h}$ post-infection (hpi) ( $\mathrm{n} \geqslant 3)$. Scale bars $=50 \mu \mathrm{m}$. IBV-Yamagata: Yamagata-like IBVs; IBV-Victoria: Victoria-like IBVs. 

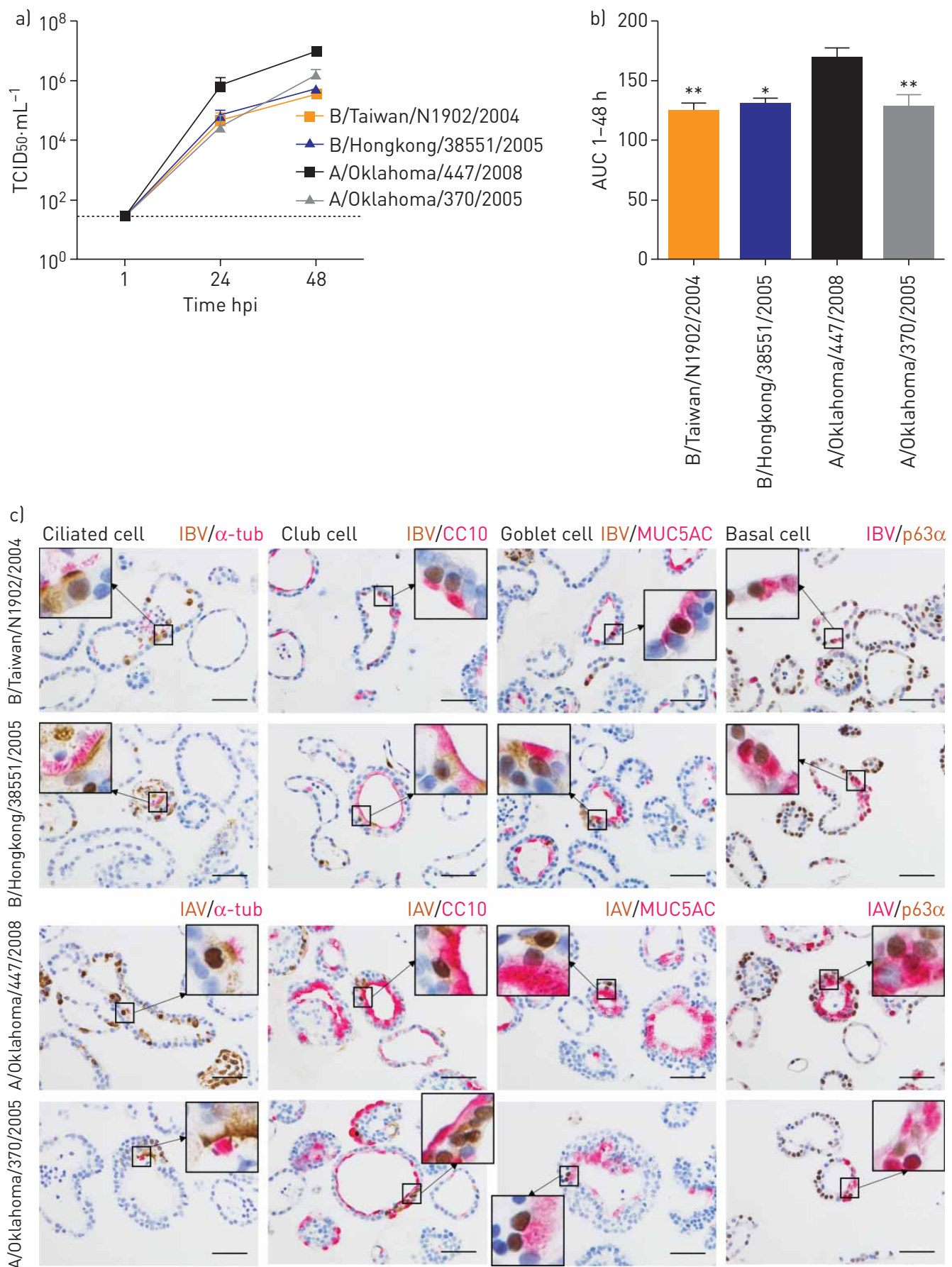

FIGURE 2 a) Replication kinetics of human seasonal influenza $A$ and $B$ viruses (IAVs and IVBs, respectively) in human airway organoids infected with $10^{6} \mathrm{TCID} 50 \cdot \mathrm{mL}^{-1}$ at $37^{\circ} \mathrm{C}$. Viral titres in culture medium (mean \pm SEM, $\mathrm{n}=3$ ) were determined by median tissue culture infective dose (TCID50) assay with a detection limit of $10^{1.5} \mathrm{TCID} 50 \cdot \mathrm{mL}^{-1}$ denoted by the dashed line. b) The total virus released from 1 to $48 \mathrm{~h}$ post-infection (hpi) was calculated as area under curve (AUC) (mean \pm SEM, $n=3$ ). Statistical significance between AUC values was analysed using one-way ANOVA with Bonferroni post-tests. *: $p<0.05$; $^{* *}$ : $\mathrm{p}<0.01$ (compared to A/Oklahoma/ 447/2008). c) Representative images of immunohistochemical double staining of virus-infected human airway organoids at 24 or $48 \mathrm{hpi}(\mathrm{n}=3$ ) for IBV antigen or IAV nucleoprotein (brown) and acetyl- $\alpha$-tubulin ( $\alpha$-tub) (pink) for ciliated cells, SCGB1A1/CC10 (CC10) (pink) for club cells, or MUC5AC (pink) for goblet cells. Basal cells were stained with p63- $\alpha$ (p63 $\alpha$ ) (brown) and IBV antigen or IAV nucleoprotein (pink). Scale bars=50 $\mu \mathrm{m}$.

effect on the ability of a virus to infect cells, we were interested to know the inhibitory effect of mucus on IBV infection. Prior to infection at MOI 2, dHBECs were either washed with PBS or not washed to remove or retain the secreted mucus, respectively. In the presence of mucus, viral titres of Yamagata-like B/Taiwan/N1902/2004, Victoria-like B/HongKong/38551/2005, A/Oklahoma/447/2008 (H1N1) and 

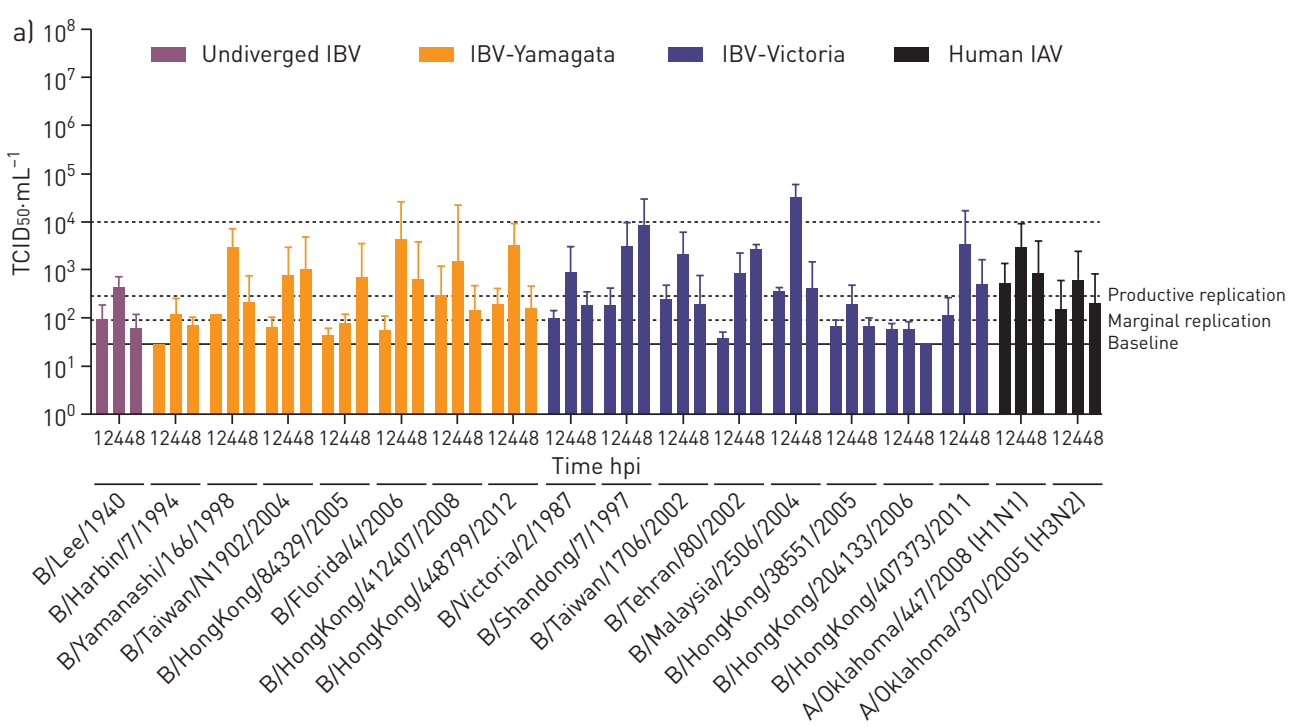

b)
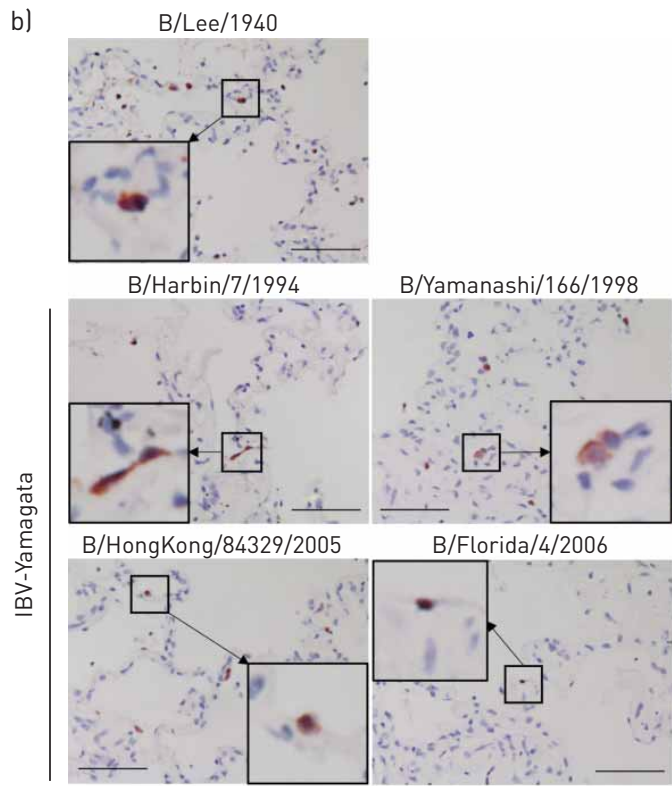

B/Taiwan/N1902/2004

$\mathrm{B} /$ Victoria/2/1987

B/Florida/4/2006

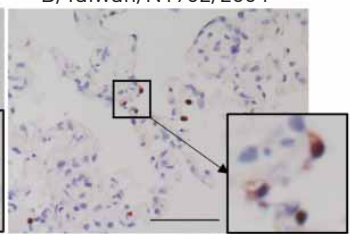

B/HongKong/412407/2008 B/HongKong/448799/2012
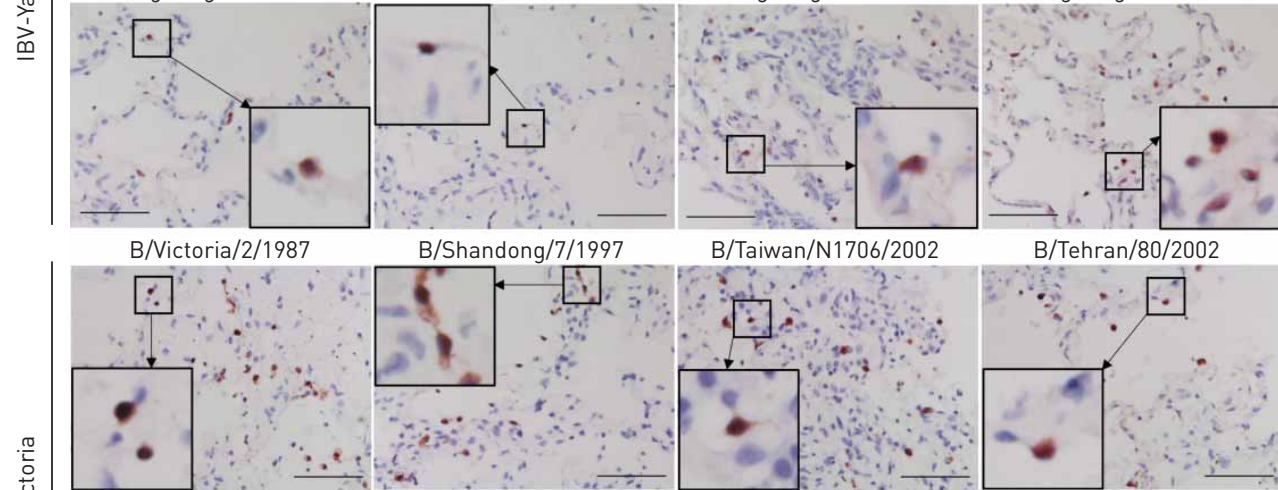

B/Shandong/7/1997

B/Taiwan/N1706/2002

B/Tehran/80/2002
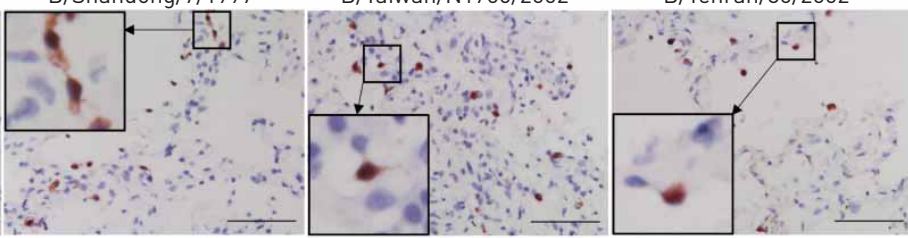

B/Malaysia/2506/2004

B/HongKong/38551/2005

B/HongKong/204133/2006

B/HongKong/407373/2011
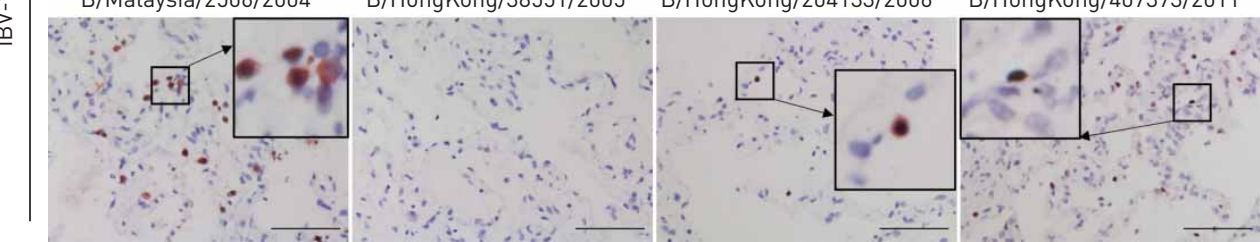

FIGURE 3 a) Replication kinetics of human seasonal influenza $A$ and $B$ viruses (IAVs and IVBs, respectively) in explant cultures of human lung infected with $\sim 10^{6} \mathrm{TCID} 50 \cdot \mathrm{mL}^{-1}$ at $37^{\circ} \mathrm{C}$. Viral titres in culture medium (mean \pm SEM, $\mathrm{n} \geqslant 3$ ) were determined by median tissue culture infective dose (TCID50) assay with a detection limit of $10^{1.5} \mathrm{TCID} 50 \cdot \mathrm{mL}^{-1}$, denoted by the baseline. Statistical significance between viral titres at different times post-infection was calculated by two-way ANOVA with Bonferroni post-tests (supplementary figure S2). b) Representative images of immunohistochemical staining for IBV nucleoprotein (brown) in virus-infected explant cultures of human lung at $48 \mathrm{~h}$ post-infection (hpi) ( $\mathrm{n} \geqslant 3)$. Scale bars $=50 \mu \mathrm{m}$. IBV-Yamagata: Yamagata-like IBVs; IBV-Victoria: Victoria-like IBVs. 
IBV-Yamagata

IBV-Victoria

Human IAV

B/Taiwan/N1902/2004

B/HongKong/38551/2005

A/Oklahoma/447/2008

B/HongKong/448799/2012

B/HongKong/407373/2011

A/Oklahoma/370/2005

Mock

a)
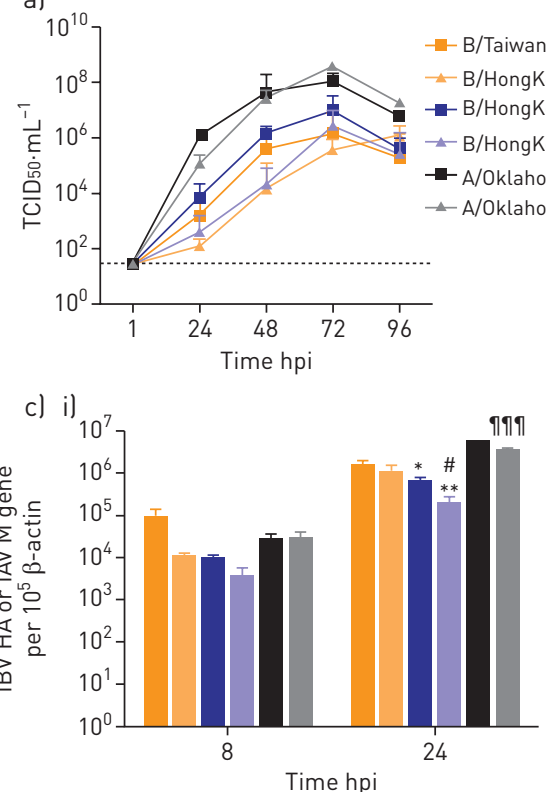

iiil

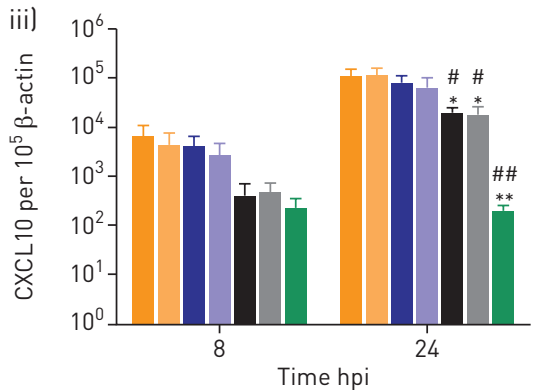

d)

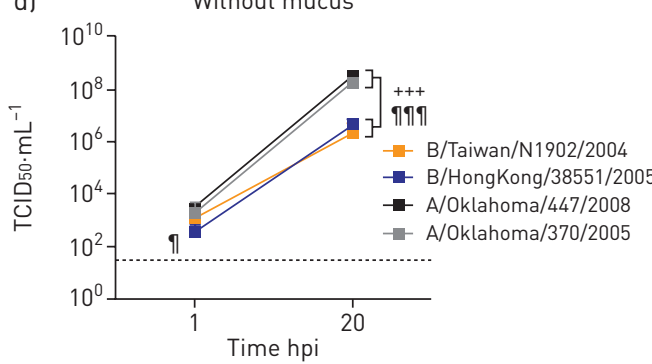

b)

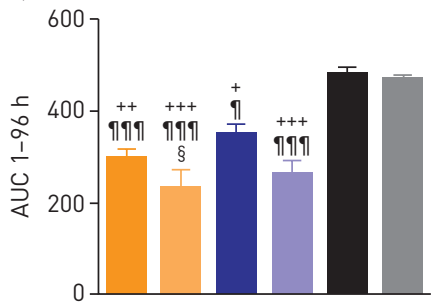

ii)
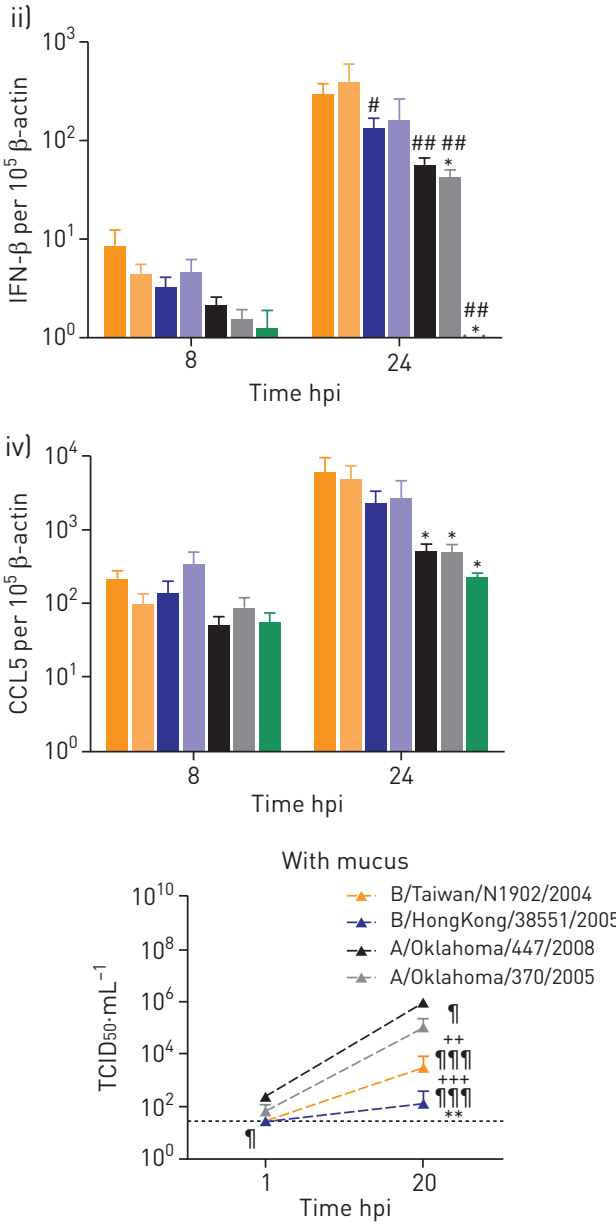

FIGURE 4 a) Replication kinetics of human seasonal influenza A and B viruses (IAVs and IVBs, respectively) in in vitro cultures of differentiated primary human bronchial epithelial cells (dHBECs) infected at multiplicity of infection (MOI) 0.01 and cultured at $37^{\circ} \mathrm{C}$. Viral titres in culture medium (mean $\pm S E M, n=4$ ) were determined by median tissue culture infective dose (TCID50) assay. b) The total virus released from 1 to $96 \mathrm{~h}$ post-infection

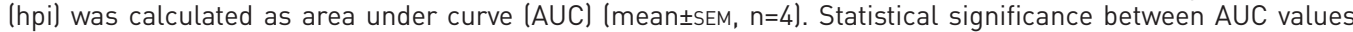
was analysed using one-way ANOVA with Bonferroni post-tests. c) Cytokine and chemokine mRNA expressions in MOl 2-infected dHBECs at 8 and $24 \mathrm{hpi}$ (mean \pm SEM, n=3). mRNA copy numbers of i) IBV haemagglutinin (HA) or IAV matrix (M), ii) interferon (IFN)- $\beta$, iii) $C-X-C$ motif chemokine (CXCL) 10 and iv) $C-C$ motif chemokine ligand (CCL)5 were expressed per $10^{5} \beta$-actin copies. d) Effect of mucus on the replication kinetics of seasonal IAVs and IBVs in dHBECs infected at MOI 2 and cultured at $37^{\circ} \mathrm{C}$. Viral titres in culture medium (mean \pm SEM, $n=3$ ) were determined by TCID50 assay at 1 and 20 hpi. Statistical significance between mRNA levels in (c) and viral titres in (d) at different times post-infection was calculated by two-way ANOVA with Bonferroni post-tests. *: p<0.05, **: p<0.01 (compared to B/Taiwan/N1902/2004); ${ }^{\#}: \mathrm{p}<0.05,{ }^{\# \#}$ : $\mathrm{p}<0.01$ (compared to B/HongKong/448799/2012); १: $p<0.05$, ๆ१ी: $p<0.001$ (compared to A/0klahoma/447/2008); ${ }^{+}: \mathrm{p}<0.05,{ }^{++}: \mathrm{p}<0.01,{ }^{+++}: \mathrm{p}<0.001$ (compared to A/Oklahoma/370/2005); ${ }^{\S}: \mathrm{p}<0.05$ (compared to B/HongKong/ 38551/2005). The detection limit of TCID50 assay was denoted by dashed lines. IBV-Yamagata: Yamagata-like IBVs; IBV-Victoria: Victoria-like IBVs. 
A/Oklahoma/370/2005 (H3N2) dropped by 2.8, 4.5, 2.5 and $3.2 \log$ TCID50. $\mathrm{mL}^{-1}$ respectively, on average, compared to cells without mucus at 20 hpi (figure $4 \mathrm{~d}$ ). Regardless of the presence of mucus, both IBVs replicated to lower titres than the seasonal IAVs. Yamagata-like B/Taiwan/N1902/2004 replicated to significantly higher titres than Victoria-like B/HongKong/38551/2005 in the presence of mucus despite similar replication without mucus, thus indicating a greater inhibitory effect of mucus on the Victoria-like IBV.

\section{IBV-induced innate immune response in primary human type-I-like AECs}

The same six-virus panel of influenza A and B viruses used in the study on dHBECs was used to investigate viral replication and induction of innate immune response in AECs. At MOI 0.01, IBVs showed productive replication but mean peak titres were only between $10^{2.5}$ and $10^{3.3} \mathrm{TCID} 50 \cdot \mathrm{mL}^{-1}$, which were $\geqslant 1 \log$ lower than those of seasonal IAVs (figure 5a). However, there was no statistically significant difference in the overall replication of IBVs and seasonal IAVs as indicated by their AUC analysis from 24 to $96 \mathrm{hpi}$ (figure 5b). At MOI 2, Victoria-like B/HongKong/407373/2011 and seasonal IAVs induced similar amount of IFN- $\beta$, CXCL10 and interleukin (IL)-29 mRNA, which were more than those induced by the other three IBVs at 24 hpi (figure $5 c$ ).

\section{IBVs predominantly bind $\alpha 2,6$-linked sialic acid receptors}

Receptor binding specificities of IBVs and IAVs were determined by their ability to agglutinate untreated and desialylated $0.5 \%$ TRBCs. All viruses, including the 16 IBVs, seasonal A/H1N1 (A/Oklahoma/447/ 2008) and A/H3N2 (A/Oklahoma/370/2005), and HPAI H5N1 (A/Hong Kong/483/1997), could agglutinate untreated TRBCs giving titres of $\geqslant 64 \mathrm{HAU}$ (haemagglutination unit) (table 2). Treatment of TRBCs with sialidase $S$, which selectively cleaves $\alpha 2,3$-linked sialic acid, did not affect agglutination of IBVs and seasonal IAVs. A predominantly $\alpha 2,3$-linked sialic acid binder HPAI H5N1 was used as a positive control of this assay, in which its haemagglutination to sialidase S-treated TRBCs was abolished as expected. Cleavage of both $\alpha 2,3$ - and $\alpha 2,6$-linked sialic acid by sialidase DAS181 prevented haemagglutination of most IBVs and IAVs, except for Yamagata-like B/HongKong/448799/2012 and $\mathrm{A} / \mathrm{H} 3 \mathrm{~N} 2$, which retained some degree of haemagglutination with four-fold drops in titres from 64 to $16 \mathrm{HAU}$. These results suggest that IBVs predominantly bind $\alpha 2,6$-linked sialic acid receptors and occasionally can bind other receptor types other than $\alpha 2,3$ - or $\alpha 2,6$-linked sialic acid.

\section{Discussion}

We have demonstrated the ability of IBVs to infect and productively replicate in explant cultures of human bronchus and lung giving viral titres comparable to those of seasonal IAVs. In human airway organoids, both IBVs and seasonal IAVs infected ciliated cells, club cells, goblet cells and basal cells demonstrating their similar tropism in the human airway. In dHBECs and AECs, some IBVs induced higher while others induced comparable to lower mRNA levels of pro-inflammatory cytokines and chemokines compared with seasonal IAVs. dHBEC-secreted mucus was shown to inhibit both IBVs and seasonal IAVs effectively.

Human conducting airway and alveolar epithelia are predominantly lined with human-like $\alpha 2,6$-linked and avian-like $\alpha 2,3$-linked sialic acid receptors, respectively [16, 17]. IBV preference for $\alpha 2,6$-linked sialic acid receptors is consistent with the higher replication of most IBVs in human bronchial than lung explants and dHBECs than AECs as reported in this study. Consistent with previous studies [18], our sialidase-treated haemagglutination assays showed IBVs predominantly bound $\alpha 2,6$-linked sialic acid receptors.

The contagiousness of seasonal influenza viruses is reflected by the millions of people infected worldwide during epidemics [19]. High infectivity and productive replication of IBVs and seasonal IAVs in human conducting airways as demonstrated in our human bronchial explants and airway organoids provide the prerequisites for their successful transmission. In contrast, HPAI H5N1 virus, which is known to have low transmissibility, infected fewer cell types and replicated poorly in these models $[10,16]$.

The prevalence of IBV infection in humans was often not as high as seasonal IAVs, although there were years when they predominated [19]. The comparable ability of IBVs and seasonal IAVs to infect and replicate in explant cultures of human conducting airways in this study suggested that differential viral fitness might not be the major contributing factor. It has been proposed that the epidemiology of IBVs and IAVs corresponds to their evolutionary behaviour and the changing herd immunity [20, 21]. Seasonal epidemics often occur due to antigenic drift. With slower mutation rates, IBVs apparently required longer time to effectively escape the existing herd immunity [20]. However, co-circulation of both Yamagata- and Victoria-like viruses have allowed reassortment of gene segments to take place between lineages, thus generating new viruses with unique gene constellations and increasing epidemic potential [20-22]. In 2001, Victoria-like IBVs re-emerged after a decade of absence in many countries with a subpopulation of 

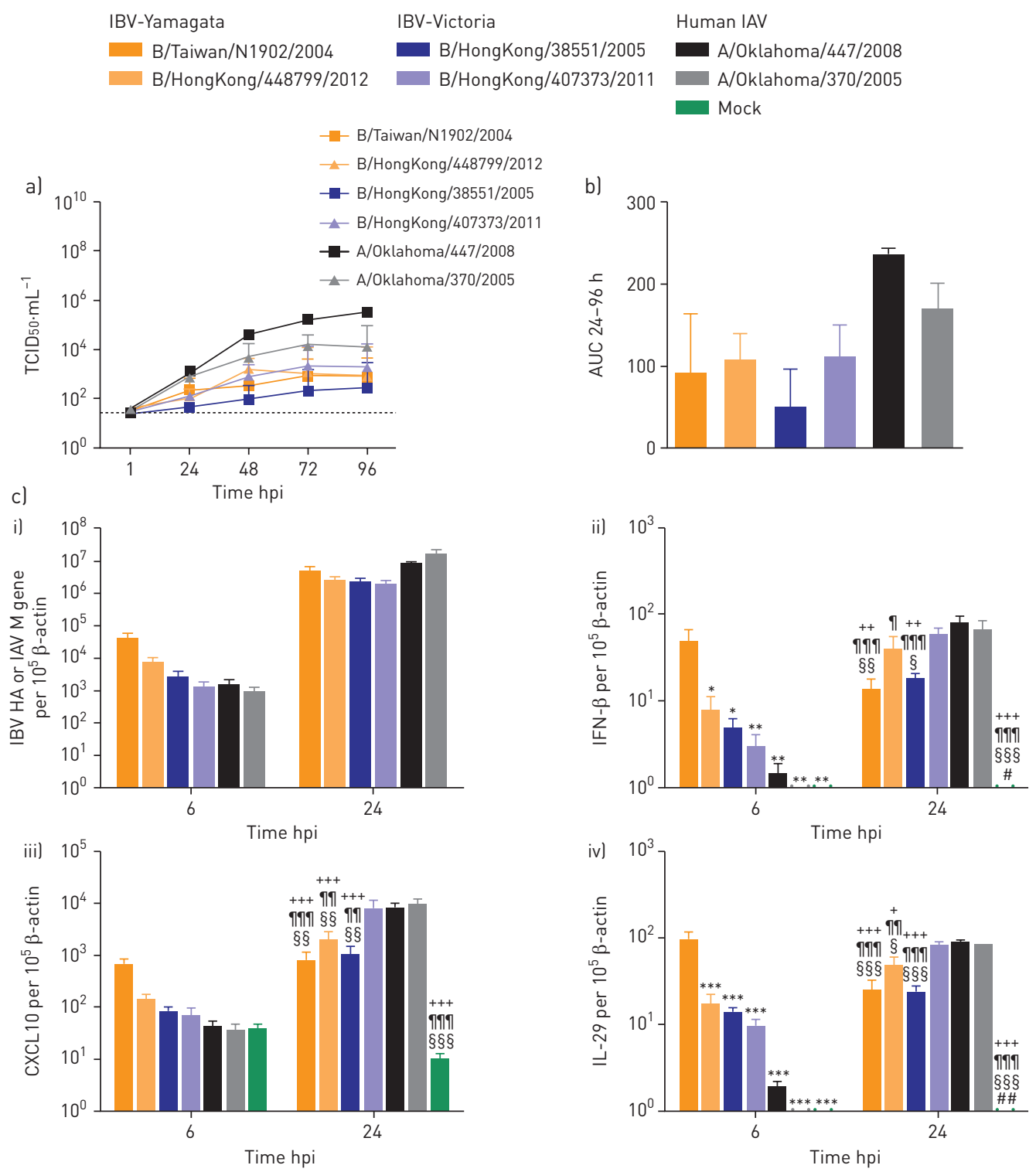

FIGURE 5 a) Replication kinetics of human seasonal influenza A and B viruses (IAVs and IVBs, respectively) in in vitro cultures of alveolar epithelial cells (AECs) infected at multiplicity of infection (MOI) 0.01 and cultured at $37^{\circ} \mathrm{C}$. Viral titres in culture medium (mean \pm SEM, $n=3$ ) were determined by median tissue culture infective dose (TCID50) assay with the detection limit denoted by a dashed line. b) The total virus released from 24 to $96 \mathrm{~h}$ post-infection (hpi) was calculated as area under curve (AUC) (mean \pm SEM, n=3). Statistical significance between AUC values was analysed using one-way ANOVA with Bonferroni post-tests. c) Cytokine and chemokine mRNA expressions in MOI 2-infected AECs at 6 and $24 \mathrm{hpi}$ (mean \pm SEM, n=3). mRNA copy numbers of i) IBV haemagglutinin (HA) or IAV matrix (M), ii) interferon (IFN)- $\beta$, iii) C-X-C motif chemokine (CXCL) 10 and iv) interleukin (IL)-29 were expressed per $10^{5} \beta$-actin copies. Statistical significance between mRNA levels at different times post-infection was calculated by two-way ANOVA with Bonferroni post-tests. *: $p<0.05$, **: $p<0.01$, ***: $p<0.001$ (compared to B/Taiwan/N1902/2004); \#: $p<0.05$, \#\#: $p<0.01$ (compared to

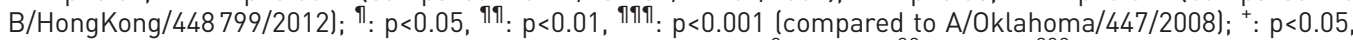
${ }^{++}: p<0.01{ }^{+++}: p<0.001$ (compared to A/Oklahoma/370/2005); ${ }^{\text {}}: p<0.05, \S \S: p<0.01, \S \S \S: p<0.001$ (compared to B/HongKong/407373/2011). IBV-Yamagata: Yamagata-like IBVs; IBV-Victoria: Victoria-like IBVs.

Victoria-like HA and Yamagata-like NA reassortant, which later spread globally and became the predominant IBV strain $[23,24]$. Such evolutionary behaviour could in turn explain the pattern of alternating dominance of the two IBV lineages. One lineage predominates until sufficient herd immunity is built against it, at which point the other lineage increases in frequency with accumulated genetic and antigenic changes [20]. Interlineage reassortment possibly resulted in strain-specific rather than lineage-specific replication and host innate immune response observed in our explant cultures of human bronchus and lung and in vitro cultures of dHBECs and AECs, respectively. Phylogenetic analysis of all gene segments revealed that viral genetic reassortment was associated with strain-specific pathogenicity of 
TABLE 2 Effects of desialylation on influenza virus haemagglutination of Turkey red blood cells (TRBCs)

\begin{tabular}{|c|c|c|c|}
\hline & \multicolumn{3}{|c|}{$0.5 \%$ TRBCs } \\
\hline & Untreated & Sialidase S & DAS181 \\
\hline \multicolumn{4}{|l|}{ Undiverged IBV } \\
\hline B/Lee/1940 & $1024^{+}$ & 1024 & 0 \\
\hline \multicolumn{4}{|l|}{ IBV-Yamagata"\# } \\
\hline B/Harbin/7/1994 & 64 & 64 & 0 \\
\hline B/Yamanashi/166/1998 & 128 & 128 & 0 \\
\hline B/Taiwan/N1902/2004 & 256 & 256 & 0 \\
\hline B/HongKong/84329/2005 & 64 & 64 & 0 \\
\hline B/Florida/4/2006 & 64 & 64 & 0 \\
\hline B/HongKong/412407/2008 & 64 & 64 & 0 \\
\hline B/HongKong/448 799/2012 & 64 & 64 & 16 \\
\hline \multicolumn{4}{|l|}{ IBV-Victoria? } \\
\hline B/Victoria/2/1987 & 64 & 64 & 0 \\
\hline B/Shandong/7/1997 & 256 & 256 & 0 \\
\hline B/Taiwan/N1706/2002 & 128 & 128 & 0 \\
\hline B/Tehran/80/2002 & 64 & 64 & 0 \\
\hline B/Malaysia/2506/2004 & 128 & 128 & 0 \\
\hline B/HongKong/38551/2005 & 128 & 128 & 0 \\
\hline B/HongKong/204 133/2006 & 64 & 64 & 0 \\
\hline B/HongKong/407373/2011 & 64 & 64 & 0 \\
\hline \multicolumn{4}{|l|}{ Human seasonal IAVs } \\
\hline A/Oklahoma/447/2008 (H1N1) & 64 & 64 & 0 \\
\hline A/Oklahoma/370/2005 (H3N2) & 64 & 64 & 16 \\
\hline \multicolumn{4}{|l|}{ HPAI H5N1 } \\
\hline A/Hong Kong/483/1997 & 512 & 0 & 0 \\
\hline
\end{tabular}

IBVs in ferrets [25]. Since humans and seals are the only natural hosts of IBVs [26], IBVs do not experience major genetic change and antigenic shift leading to pandemics and highly pathogenic strains like IAVs [21].

We did not observe any huge replication difference among IBV strains in human lung explants. Most had replication efficiencies comparable with seasonal IAVs. Moreover, IBVs, like seasonal IAVs, were low pro-inflammatory cytokine and chemokine inducers. They are apparently not as pathogenic as HPAI H5N1, which has strong tropism for human lung and ability to induce cytokine dysregulation [27, 28]. However, their virulence should not be neglected, especially for people with lower immunity like the elderly, young children and those with underlying diseases, as pneumonia, ARDS and death were more often associated with IBV infection in these groups $[6,29]$. In the US, $22-44 \%$ of paediatric influenza deaths each season were attributable to influenza B between 2004 and 2011, excluding the 2009-2010 pandemic [30]. However, treatment options for IBV infection are limited, with oseltamivir and zanamivir being the only two drugs approved by the US Food and Drug Administration [31]. Yet oseltamivir is less effective against IBVs than IAVs and zanamivir is not prescribed to children aged $<7$ years. Recent availability of quadrivalent influenza vaccines, which include IBVs from both lineages, may give broader protection and reduce the burden of IBV-lineage mismatch often encountered by trivalent vaccines [32]. Nevertheless, evidence has accumulated on the sporadic transmission of IBVs from humans to pigs indicating potential expansion of host species and increasing risks [33]. Our ongoing risk assessment had suggested that swine respiratory tract is susceptible to IBV infection (unpublished data). Continuous surveillance and risk assessment of newly evolving IBV strains are therefore necessary.

The large numbers of mucins in mucus contain high content of terminal sialic acid which could act as "decoy" receptors to trap viruses and prevent them from infecting cell surfaces [34]. ZANIN et al. [35] observed a similar inhibitory effect of dHBEC-secreted mucus on swine pandemic and human A/H1N1 as on IBVs and seasonal IAVs demonstrated in this study. Additionally, they showed IAVs with higher NA enzymatic activity penetrated mucus layer better than their counterparts with less balanced HA-NA 
activities. We speculated similar mechanisms for the greater inhibition of the Victoria-like IBV than Yamagata-like IBV in our study. However, further investigation is required and may promote better understanding in IBV transmissibility.

Consistent with previous histology studies on the similar organisation, composition and cellular diversity of human airway organoids and dHBECs with human airways $[10,14,36]$, the replication kinetics of the tested IBVs and seasonal IAVs in human bronchial explants, human airway organoids and dHBECs were fairly comparable. While using human bronchial explants would be ideal since they are the most original, there is limited availability, donor-to-donor variability and short-term viability of $48-72 \mathrm{~h}$ of such tissues. Although dHBECs can be expanded and cultured for longer periods, they normally have poor stability and reproducibility beyond passage three [37]. In contrast, human airway organoids can be rescued from long-term cryopreservation and expanded up to at least passage 19 without much alteration [10, 36]. These features allow organoids of the same donors, healthy or diseased, to be used repeatedly in risk assessments of different viruses as they emerge over time and their results compared [10]. Another important advantage is that organoids can be genetically engineered to study gene functions in disease models [38]. Moreover, there is a continuous cell structure in the three-dimensional spheres of human airway organoids that should allow better study of mucus movement and ciliary beating in relations to mucociliary clearance during influenza virus infections, as compared to the interrupted cell layers in human bronchial explants and dHBECs.

Comparable viral replication in human airway organoids and bronchial explants for the tested IBVs and seasonal IAVs allow anticipation of similar replication efficiencies in organoids as in human bronchial explants for the other IBVs. Previously, IAVs with different replication kinetics in human bronchial explants, including human 2009 pandemic H1N1, H7N9, HPAI H5N1 and HPAI H5N6, also showed similar replication competence in human airway organoids [10].

We chose the two Oklahoma seasonal IAVs to compare with IBVs in this study since they had been well characterised with glycan arrays and showed good replication in human bronchial and lung explants [39, 40]. Furthermore, their replication kinetics did not show major differences from those of the two Hong Kong seasonal IAVs (supplementary figure S3).

In conclusion, our study of IBVs isolated from 1940 to 2012 clearly indicated the ability of IBVs to infect and productively replicate in a variety of cell types in the human respiratory tract epithelium. There was higher preference for the conducting airway than the lower lung and strain-specific rather than lineage-specific pathogenicity. These highlighted the non-negligible virulence of IBVs which require more attention and investigation to alleviate the disease burden, especially when treatment options are limited.

Acknowledgements: We would like to acknowledge I.J. Su (Taiwan National Cheng Kung University, Taiwan), Vicki Gregory (WHO Collaborating Centre for Influenza, National Institute for Medical Research, London, UK) and K.H. Chan (Dept of Microbiology, The University of Hong Kong, Hong Kong SAR, China) for their generous sharing of the influenza B virus seed stock. We thank the International Reagent Resource, Centers for Disease Control and Prevention for providing us with the WHO monoclonal antibody and Kevin Fung (Dept of Pathology, The University of Hong Kong) for his technical assistance in immunohistochemistry. Our special thanks to Arnaud Orelle, a former member of our group, who was involved in the early stages of the study.

Conflict of interest: C.H.T. Bui has nothing to disclose. M.M.T. Ng has nothing to disclose. M.C. Cheung has nothing to disclose. K-C. Ng has nothing to disclose. M.P.K. Chan has nothing to disclose. L.L.Y. Chan has nothing to disclose. J.H.M. Fong has nothing to disclose. J.M. Nicholls has nothing to disclose. J.S.M. Peiris reports grants from the Research Grants Council of the Hong Kong SAR and the US National Institute of Allergy and Infectious Diseases (NIAID), during the conduct of the study. R.W.Y Chan reports grants from the Research Fund Secretariat, Food and Health Bureau, Hong Kong Special Administrative Region, during the conduct of the study. M.C.W. Chan reports grants from the Research Grants Council of the Hong Kong SAR and the US National Institute of Allergy and Infectious Diseases (NIAID), during the conduct of the study.

Support statement: This work was supported by the Health and Medical Research Fund (Ref: 13120772), Research Fund Secretariat, Food and Health Bureau, Hong Kong Special Administrative Region; the Theme Based Research Scheme (T11-705/14N), the Research Grants Council of the Hong Kong SAR; and the US National Institute of Allergy and Infectious Diseases (NIAID) under Centers of Excellence for Influenza Research and Surveillance (CEIRS) contract (HHSN27220140006C). Funding information for this article has been deposited with the Crossref Funder Registry.

\section{References}

Francis T, Jr. A new type of virus from epidemic influenza. Science 1940; 92: 405-408.

Bouvier NM, Palese P. The biology of influenza viruses. Vaccine 2008; 26: Suppl. 4, D49-D53.

Rota PA, Wallis TR, Harmon MW, et al. Cocirculation of two distinct evolutionary lineages of influenza type B virus since 1983. Virology 1990; 175: 59-68.

4 Lin YP, Gregory V, Bennett M, et al. Recent changes among human influenza viruses. Virus Res 2004; 103: 47-52.

5 Thompson WW, Shay DK, Weintraub E, et al. Influenza-associated hospitalizations in the United States. JAMA 2004; 292: 1333-1340. 
6 Chiu SC, Lin JH, Wang HC, et al. Molecular epidemiologic and clinical characteristics of influenza B-associated complications among hospitalized patients during an outbreak in Taiwan. Int J Infect Dis 2014; 23: 94-100.

7 Matias G, Taylor R, Haguinet F, et al. Estimates of mortality attributable to influenza and RSV in the United States during 1997-2009 by influenza type or subtype, age, cause of death, and risk status. Influenza Other Respir Viruses 2014; 8: 507-515.

8 Chiu SS, Chan KH, So LY, et al. The population based socioeconomic burden of pediatric influenza-associated hospitalization in Hong Kong. Vaccine 2012; 30: 1895-1900.

9 World Health Organization. Tool for Influenza Pandemic Risk Assessment. 2016. http://apps.who.int/iris/ bitstream/handle/10665/250130/WHO-OHE-PED-GIP-2016.2-eng.pdf?sequence=1

10 Hui KPY, Ching RHH, Chan SKH, et al. Tropism, replication competence, and innate immune responses of influenza virus: an analysis of human airway organoids and ex vivo bronchus cultures. Lancet Respir Med 2018; 6: $846-854$

11 Chan MC, Chan RW, Chan LL, et al. Tropism and innate host responses of a novel avian influenza A H7N9 virus: an analysis of ex vivo and in vitro cultures of the human respiratory tract. Lancet Respir Med 2013; 1: 534-542.

12 Chan LL, Bui CT, Mok CK, et al. Evaluation of the human adaptation of influenza A/H7N9 virus in PB2 protein using human and swine respiratory tract explant cultures. Sci Rep 2016; 6: 35401.

13 Chan MC, Chan RW, Yu WC, et al. Tropism and innate host responses of the 2009 pandemic H1N1 influenza virus in ex vivo and in vitro cultures of human conjunctiva and respiratory tract. Am J Pathol 2010; 176: 1828-1840.

14 Chan RW, Yuen KM, Yu WC, et al. Influenza H5N1 and H1N1 virus replication and innate immune responses in bronchial epithelial cells are influenced by the state of differentiation. PLoS One 2010; 5: e8713.

15 Fahy JV, Dickey BF. Airway mucus function and dysfunction. N Engl J Med 2010; 363: 2233-2247.

16 Shinya K, Ebina M, Yamada S, et al. Avian flu: influenza virus receptors in the human airway. Nature 2006; 440; 435-436.

17 Nicholls JM, Bourne AJ, Chen H, et al. Sialic acid receptor detection in the human respiratory tract: evidence for widespread distribution of potential binding sites for human and avian influenza viruses. Respir Res 2007; 8: 73.

18 Wang YF, Chang CF, Chi CY, et al. Characterization of glycan binding specificities of influenza B viruses with correlation with hemagglutinin genotypes and clinical features. J Med Virol 2012; 84: 679-685.

19 World Health Organization. Seasonal Influenza Reviews: Archives 2010-2016. www.who.int/influenza/ surveillance_monitoring/updates/GIP_surveillance_summary_reviews_archives/en/

20 Chen R, Holmes EC. The evolutionary dynamics of human influenza B virus. J Mol Evol 2008; 66: 655-663.

21 McCullers JA, Wang GC, He S, et al. Reassortment and insertion-deletion are strategies for the evolution of influenza B viruses in nature. J Virol 1999; 73: 7343-7348.

22 Lindstrom SE, Hiromoto $\mathrm{Y}$, Nishimura $\mathrm{H}$, et al. Comparative analysis of evolutionary mechanisms of the hemagglutinin and three internal protein genes of influenza B virus: multiple cocirculating lineages and frequent reassortment of the NP, M, and NS genes. J Virol 1999; 73: 4413-4426.

23 Shaw MW, Xu X, Li Y, et al. Reappearance and global spread of variants of influenza B/Victoria/2/87 lineage viruses in the 2000-2001 and 2001-2002 seasons. Virology 2002; 303: 1-8.

$24 \mathrm{Xu} \mathrm{X}$, Lindstrom SE, Shaw MW, et al. Reassortment and evolution of current human influenza A and B viruses. Virus Res 2004; 103: 55-60.

25 Huang SS, Banner D, Paquette SG, et al. Pathogenic influenza B virus in the ferret model establishes lower respiratory tract infection. J Gen Virol 2014; 95: 2127-2139.

26 Osterhaus AD, Rimmelzwaan GF, Martina BE, et al. Influenza B virus in seals. Science 2000; 288: 1051-1053.

27 Yu WC, Chan RW, Wang J, et al. Viral replication and innate host responses in primary human alveolar epithelial cells and alveolar macrophages infected with influenza H5N1 and H1N1 viruses. J Virol 2011; 85: 6844-6855.

28 Uiprasertkul M, Puthavathana P, Sangsiriwut K, et al. Influenza A H5N1 replication sites in humans. Emerg Infect Dis 2005; 11: 1036-1041.

29 Ambrose CS, Levin MJ. The rationale for quadrivalent influenza vaccines. Hum Vaccin Immunother 2012; 8: 81-88.

30 Glezen WP, Schmier JK, Kuehn CM, et al. The burden of influenza B: a structured literature review. Am J Public Health 2013; 103: e43-e51.

31 Burnham AJ, Baranovich T, Govorkova EA. Neuraminidase inhibitors for influenza B virus infection: efficacy and resistance. Antiviral Res 2013; 100: 520-534.

32 Gemmill I, National Advisory Committee on Immunization. Summary of the National Advisory Committee on Immunization (NACI) Statement on Seasonal Influenza Vaccine for 2015-2016. Can Commun Dis Rep 2015; 41: 227-232.

33 Ran Z, Shen H, Lang Y, et al. Domestic pigs are susceptible to infection with influenza B viruses. J Virol 2015; 89: 4818-4826.

34 Zanin M, Baviskar P, Webster R, et al. The interaction between respiratory pathogens and mucus. Cell Host Microbe 2016; 19: 159-168.

35 Zanin M, Marathe B, Wong SS, et al. Pandemic Swine H1N1 influenza viruses with almost undetectable neuraminidase activity are not transmitted via aerosols in ferrets and are inhibited by human mucus but not swine mucus. I Virol 2015; 89: 5935-5948.

36 Sachs N, Papaspyropoulos A, Zomer-van Ommen DD, et al. Long-term expanding human airway organoids for disease modeling. EMBO J 2019; 38: e100300.

37 Reeves SR, Barrow KA, White MP, et al. Stability of gene expression by primary bronchial epithelial cells over increasing passage number. BMC Pulm Med 2018; 18: 91.

38 Chen YW, Huang SX, de Carvalho A, et al. A three-dimensional model of human lung development and disease from pluripotent stem cells. Nat Cell Biol 2017; 19: 542-549.

39 Walther T, Karamanska R, Chan RW, et al. Glycomic analysis of human respiratory tract tissues and correlation with influenza virus infection. PLoS Pathog 2013; 9: e1003223.

40 Kumari K, Gulati S, Smith DF, et al. Receptor binding specificity of recent human H3N2 influenza viruses. Virol 2007; 4: 42. 